\section{Norrin vs. VEGF}

\section{By Lev Osherovich, Senior Writer}

Two papers in Cell describe a new signaling pathway that acts upstream of VEGF to control vascular development in the embryonic retina. The pathway is also present in adults, but the question is whether it's actually active and thus potentially targetable in diseases of retinal neovascularization, including diabetic retinopathy, retinopathy of prematurity and potentially wet age-related macular degeneration.

One study, from a team at The Johns Hopkins University School of Medicine, establishes how a secreted proangiogenic protein called Norrie disease pseudoglioma (NDP; Norrin) interacts with its receptor, frizzled homolog 4 (FZD4), to enable vascular growth during embryonic development. ${ }^{1}$ The other study, from a team at the Genentech Inc. unit of Roche, identifies an additional protein at the cell surface, tetraspanin 12 (TSPAN12), that helps FZD4 bind to Norrin. ${ }^{2}$

"The main finding is that the Norrin system is a novel signaling pathway with substantial effects on vascular development in mice and humans," said Jeremy Nathans, professor of molecular biology and genetics at Johns Hopkins and the lead author of one of the studies.

FZD4 is a transmembrane receptor present throughout the body during development and adulthood. In most tissues it responds to Wnt, a morphogenic protein that promotes cell division. Norrin is an alternative ligand for FZD4 present only in the retina and inner ear.

Norrin, FZD4 and TSPAN12 act together primarily in the retina and could thus be better targets for retina-specific anti-neovascular therapies than VEGF, which is active throughout the body. As transmembrane proteins, FZD4 and TSPAN12 are also potentially amenable to orally administered systemic small molecule therapeutics.

A fourth protein, low-density lipoprotein receptor-related protein 5 (LRP5), is part of the FZD4-TSPAN12 receptor complex. LRP5 knockouts appear to behave similarly to FZD4 knockouts.

\section{The eyes have it}

Previously, Norrin and FZD4 have been linked to defects in retinal vascular development that lead to rare hereditary forms of blindness in humans. However, it was not known specifically which retinal tissues required these proteins because the retina consists of complex layers of endothelial and nervous cells.

To tackle the question, the Nathans team designed knockouts of Fzd4, the mouse homolog of FZD4, and knock-ins of a Norrin reporter gene that allowed them to sort out which protein acted where.

The group found that Fzd4 was present throughout the body, including on the surface of retinal endothelial cells, where it was essential for normal eye development. Selective knockout of $F z d 4$ in those cells resulted in mice that had underdeveloped retinal vasculature and vision deficits but were otherwise normal.

Nathans suggested that the effects of $F z d 4$ knockout could be specific to the eye because other Frizzled homologs make up for the receptor's absence outside the retina.

The team also found that Norrin was secreted by the glial cells of the retina and that this precise localization of Norrin production was needed for normal vascular development. Mouse embryos that overexpressed Norrin in other tissues developed disorganized vasculature throughout the body and did not survive to birth.

Nathans thus thinks the Norrin system plays a role in the early steps of vascular development, helping to shape capillaries by cementing interactions between the various cell types of the vasculature and retina.

In addition, Nathans suspects that Norrin signaling during early vascular development, and possibly also during neovascularization, sets the stage for later activity by VEGF. As a result, he said, VEGF is unlikely to have an effect in the absence of Norrin.

"You'd think that the VEGF system would trump other regulatory systems, but that actually doesn't happen. The view of VEGF as the master regulator that leads to vascular growth may not be correct," he said. "At least in the context of retinal vascularization, the VEGF system cannot override the Norrin system."

\section{Spanning the gap}

The Genentech study team, led by senior scientist Weilan Ye, added to the list of players in the Norrin system. TSPAN12 belongs to a family of plasma membrane proteins called tetraspanins. Other members of this family previously have been linked to retinal diseases. ${ }^{3}$

"We have found a new component of the Norrin signal transduction pathway-TSPAN12," said Ye. "Our study adds mechanistic insights into how FZD4 transduces Norrin signal"

Ye's team discovered Tspan 12 while searching for mouse mutants with abnormal vascular phenotypes. Tspan 12 knockout mice had aberrant retinal vascular development that was similar to Norrin, Lrp5 and Fzd4 knockouts.

Using mice with Norrin, Lrp5 and Tspan 12 mutations, the researchers found these two proteins worked in the same pathway.

Cell culture studies showed that Tspan 12 physically interacted with Fzd4 and increased the receptor's ability to respond to Norrin. Overexpression of human TSPAN12 in cultured cells overcame signaling defects caused by mutations in Norrin and FZD4. 
Ye thinks that TSPAN12's ability to enhance FZD4 activity could explain why the Norrin-FZD4 pathway is active only in the retinal blood vessels, even though FZD4 is present in many cell types in the eye.

\section{Wary of Norrin}

The biggest question about modulating the Norrin pathway for therapeutic purposes is whether it plays a role in pathological neovascularization in the adult retina.

"We don't know if this pathway will be targetable for retinal disease of adulthood," said Ye. "Potentially, this pathway could be involved in degenerative retinal diseases, but right now there's nothing in the literature about this."

"We know that components of the pathway are present in the mature retina, so it's possible that dysregulation of this pathway is part of the picture" in diseases like diabetic retinopathy, said Nathans.

Nathans noted that the prospects for tar-

"Blockade of these targets even in adults should markedly reduce neovascularization but could potentially be associated with alteration of the normal retinal vasculature," said Pruneau.

Fovea, which is being acquired by sanofi-aventis Group, is developing compounds to treat back-of-the-eye diseases. FOV2302, a plasma kallikrein-kinin inhibitor, is in Phase IIa testing to treat retinal vein occlusion (RVO)-induced acute macular edema. FOV2501, an intravitreal formulation of rod-derived cone viability factor (RdCVF), is in preclinical development to treat retinitis pigmentosa.

Because Norrin, FZD4 and TSPAN12 all have extracellular domains, "a screening assay for agonists or antagonists can likely be set up," Pruneau noted.

Nathans and Genetech's Ye both noted that although certain aspects of retinal neovascular disease can be studied in mice, it's difficult to recapitulate the whole progression of diabetic retinopathy and AMD in rodents.

"Experimental approaches in nonrodent spegeting the Norrin pathway in wet age-related macular degeneration (AMD), the most common retinal neovascular disease, are less certain than for other indications. In wet AMD, abnormal blood vessels originate from the choroid layer, a different part of the eye than the retinal endothelium cells on which his team is focused.

"It's an open question about how effective manipulating the Norrin system would be in wet AMD," he said.

Ryo Kubota, chairman, president and CEO of Acucela Inc., said the new targets are tempting in multiple ophthalmic conditions, including wet AMD, but much more preclinical work is needed.

"Lucentis works only in about $30 \%$ of patients," said Kubota, and "these targets might address the rest." However, he wanted to know whether any of the Norrin pathway components are "overexpressed in a disease situation."

Lucentis ranibizumab, a mAb from Genentech and Novartis AG that targets VEGF-A, is marketed for wet AMD. Lucentis also is in Phase III trials for diabetic edema, a condition that often precedes diabetic retinopathy.

Acucela is targeting the visual cycle, a metabolic process in the retina that leads to oxidative stress and retinal neovascularization. Acucela's ACU-4429 visual cycle inhibitor is being co-developed with Otsuka Pharmaceutical Co. Ltd. and has completed Phase I testing.

Didier Pruneau, head of scientific operations at Fovea Pharmaceuticals S.A., said earlier studies have suggested that the Norrin pathway is probably active in the adult retina. Nevertheless, because the pathway seems to be so central to retinal vascularization, Pruneau was concerned about the potentially severe side effects of antagonizing it. cies are needed," said Pruneau. "There is a long way to go prior to getting into clinical trials."

Ye's next task is to identify agonists and antagonists of the NorrinFZD4 pathway. She also plans to test whether TSPAN12 plays a role in solid tumors, in which neovascularization helps to feed growing cancers.

Nathans, meanwhile, plans to test the effect of knocking down Norrin in mice with hypoxic retinas, a standard model of retinal neovascular diseases.

Nathans did not patent his discoveries. Genentech did not disclose whether it has IP related to Ye's discoveries.

Osherovich, L. SciBX 2(43); doi:10.1038/scibx.2009.1589

Published online Nov. 5, 2009

\section{REFERENCES}

1. Ye, X. et al. Cell; published online Oct. 16, 2009; doi:10.1016/j.cell.2009.07.047

Contact: Jeremy Nathans, The Johns Hopkins University School of Medicine, Baltimore, Md.

e-mail: jnathans@jhmi.edu

2. Junge, J. et al. Cell; published online Oct. 16, 2009;

doi:10.1016/j.cell.2009.07.048

Contact: Weilan Ye, Genentech Inc., South San Francisco, Calif. e-mail: loni@gene.com

3. Hemler, M.E. Nat. Rev. Mol. Cell Biol. 6, 801-811 (2005)

\section{COMPANIES AND INSTITUTIONS MENTIONED}

Acucela Inc., Bothell, Wash.

Genentech Inc., South San Francisco, Calif.

Fovea Pharmaceuticals S.A., Paris, France

The Johns Hopkins University School of Medicine, Baltimore, Md. Novartis AG (NYSE:NVS; SIX:NOVN), Basel, Switzerland

Otsuka Pharmaceutical Co. Ltd. (Tokyo:4768), Tokyo, Japan sanofi-aventis Group (Euronext:SAN; NYSE:SNY), Paris, France Roche (SIX:ROG; OTCQX:RHHBY), Basel, Switzerland 